\title{
MATRICES WITH PRESCRIBED ENTRIES AND EIGENVALUES. I 1
}

\author{
G. N. DE OLIVEIRA
}

\begin{abstract}
It is proved that there exists a matrix (over an appropriate field) with prescribed eigenvalues and a prescribed nonprincipal diagonal. It is also proved that there exists an $\boldsymbol{n} \times \boldsymbol{n}$ matrix, over an arbitrary field, with prescribed eigenvalues and with $n-1$ prescribed arbitrary entries.
\end{abstract}

In an $n \times n$ matrix consider the positions $(i, \sigma(i)), i=1, \cdots, n$, for some permutation $\sigma$. This set of positions is called the $\sigma$-diagonal. A $\sigma$-diagonal is called principal if and only if $\sigma$ is the identity permutation.

Many papers on matrices with prescribed characteristic polynomial (or eigenvalues) and prescribed entries have been written ([1], [3], [4], [7], [8], [9]). It is well known that there exists an $n \times n$ matrix, over an arbitrary field, with prescribed principal diagonal and characteristic polynomial $f(\lambda)=\lambda^{n}-c_{1} \lambda^{n-1}+\cdots$ if and only if the sum of the prescribed entries equals $c_{1}$ [1]. In the present paper we shall extend this result (Theorems 1 and 2 below) for the case in which the prescribed diagonal is not principal. In Theorem 3 we generalize for an arbitrary field a theorem in [3]. Our proof is much simpler than that of [3] and gives an effective method for constructing the matrix.

Let $\sigma$ be a permutation of $(1, \cdots, n)$ different from the identity permutation. We wish to prescribe the entries in the positions $(i, \sigma(i)), i=1, \cdots, n$, of an $n \times n$ matrix. As in [3] let $\sigma=\sigma_{1} \cdots \sigma_{s}$ be the representation of $\sigma$ as the product of the disjoint cycles $\sigma_{k}$ and $n_{k}$ be the length of $\sigma_{k}$. A permutation on the rows of a matrix followed by the same permutation on the columns does not alter the eigenvalues (we also wish to prescribe the eigenvalues). Hence, applying an appropriate permutation, we may assume, without loss of generality, that

$$
\begin{aligned}
\sigma_{1}=\left(1, \cdots, n_{1}\right), \sigma_{2}=\left(n_{1}+1, \cdots,\right. & \left.n_{1}+n_{2}\right), \cdots, \\
& \sigma_{s}=\left(n_{1}+\cdots+n_{s-1}+1, \cdots, n\right)
\end{aligned}
$$

Received by the editors February 29, 1972.

AMS (MOS) subject classifications (1970). Primary 15A18.

1 This research was supported by the C. Gulbenkian Foundation.

(C) American Mathematical Society 1973 
and that $n_{i}>2$ for $i=1, \cdots, \alpha, n_{i}=2$ for $i=\alpha+1, \cdots, \beta, n_{i}=1$ for $i=$ $\beta+1, \cdots, s$. Of course some of the numbers $\alpha, \beta-\alpha, s-\beta$ may be zero but we assume that $\alpha$ and $\beta-\alpha$ do not vanish simultaneously, since otherwise $\sigma$ would be the identity permutation.

Now we state our results.

THEOREM 1. Suppose that at least one of the cycles $\sigma_{1}, \cdots, \sigma_{s}$ has length $>2$. Let $\Phi$ be an arbitrary field and $\lambda_{1}, \cdots, \lambda_{n}, a_{1}, \cdots, a_{n} \in \Phi$. There exists an $n \times n$ matrix over $\Phi$ with eigenvalues $\lambda_{1}, \cdots, \lambda_{n}$ and $a_{i}$ in the position $(i, \sigma(i)), i=1, \cdots, n$.

If there is no cycle of length $>2$, a similar result can be proved but a restriction is necessary.

THEOREM 2. Suppose $\sigma_{1}, \cdots, \sigma_{\beta}$ are all of length 2 , i.e., $\sigma_{i}=(2 i-1,2 i)$, $i=1, \cdots, \beta$. Let $\lambda_{1}, \cdots, \lambda_{n}, a_{1}, \cdots, a_{n}$ be elements of the field $\Phi$. Suppose that there is one value $i_{0}(\leqq \beta)$ of $i$ such that the equation

$$
x^{2}-(\Theta+\rho) x+a_{2 i_{0}-1} a_{2 i_{0}}+\Theta \rho=0,
$$

where $\Theta, \rho$ are two of the $\lambda_{k}$, has a solution in $\Phi$. Then there exists an $n \times n$ matrix over $\Phi$ with eigenvalues $\lambda_{1}, \cdots, \lambda_{n}$ and $a_{j}$ in the position $(j, \sigma(j)), j=1, \cdots, n$.

REMARK. The restriction imposed in the hypothesis of this theorem cannot, in general, be removed. Example: if $n=2$ and $x^{2}-\left(\lambda_{1}+\lambda_{2}\right) x+$ $a_{1} a_{2}+\lambda_{1} \lambda_{2}=0$ has no solution in $\Phi$, there exists no $2 \times 2$ matrix over $\Phi$ with $\lambda_{1}, \lambda_{2}$ as eigenvalues and $a_{1}, a_{2}$ in the positions $(1,2)$ and $(2,1)$ respectively.

In these two theorems we prescribe a nonprincipal diagonal. In the following we prescribe only $n-1$ entries but they need not belong to a diagonal.

THEOREM 3. Let $\Phi$ be an arbitrary field and $\lambda_{1}, \cdots, \lambda_{n}, a_{1}, \cdots$, $a_{n-1} \in \Phi$. Let $\left(i_{1}, j_{1}\right), \cdots,\left(i_{n-1}, j_{n-1}\right)$ be $n-1$ arbitrary but distinct positions in an $n \times n$ matrix. There exists an $n$-square matrix over $\Phi$ with eigenvalues $\lambda_{1}, \cdots, \lambda_{n}$ and $a_{t}$ in the position $\left(i_{t}, j_{t}\right), t=1, \cdots, n-1$.

The proofs will be carried out by means of the $L$-transformation we introduced in [5] for constructing stochastic matrices with prescribed eigenvalues. Since [5] has been published in Portuguese we shall define the $L$-transformation (indeed only the particular case we need here) before proceeding to the proofs. 
We denote by $I_{k}^{(i, j)}, i \neq j, i, j \leqq k$, the matrix obtained from the $k \times k$ identity matrix by adding its $i$ th row to its $j$ th row. The inverse of $I_{k}^{(i, j)}$ is obtained from $I_{k}^{(i, j)}$ by replacing its only off diagonal 1 by -1 . If $A$ is a $k \times k$ matrix,

$$
I_{k}^{(i, j)} A I_{k}^{(i, j)^{-1}}
$$

is easily constructed by adding the $i$ th row of $A$ to its $j$ th row and subtracting its $j$ th column from its $i$ th column. Let $\Phi_{r}$ denote the set of all $r \times r$ matrices over the field $\Phi$. Let $C$ and $X$ be respectively $s \times s$ and $m \times s$ fixed matrices, both over $\Phi$. We define an operator $L_{i j n}^{(X, C)}: \Phi_{m} \rightarrow \Phi_{n}$, $n=m+s$, as follows. Let $A \in \Phi_{m}$ and $B=\left[\begin{array}{ll}A & X \\ 0 & C\end{array}\right]$, where 0 is the $s \times m$ zero block. We put

$$
L_{i j n}^{(X, C)} A=I_{n}^{(i, j)} B I_{n}^{(i, j)^{-1}}
$$

The matrix so obtained is called the $L$-transform (or, more rigourously, the $L_{i j n}^{(X, C)}$-transform) of $A$. Clearly the characteristic polynomial of $L_{i j n}^{(X, C)} A$ is the product of the characteristic polynomials of $A$ and $C$, i.e., the eigenvalues of $L_{i j n}^{(X . C)} A$ are those of $A$ together with those of $C$.

Proof of Theorem 1 . We have

$$
\sigma_{i}=\left(n_{1}+\cdots+n_{i-1}+1, \cdots, n_{1}+\cdots+n_{i}\right)
$$

and the length $n_{i}$ of $\sigma_{i}$ for $i=1, \cdots, \alpha$ is $>2$. Let $A_{i}, i \leqq \alpha$, be the $\left(n_{i}-1\right) \times$ $\left(n_{i}-1\right)$ matrix having $\lambda_{n_{1}+\cdots+n_{i-1}+1}, \cdots, \lambda_{n_{1}+\cdots+n_{i}-1}$ down the principal diagonal, $a_{n_{1}+\cdots+n_{i-1}+1}, \cdots, a_{n_{1}+\cdots+n_{i}-2}$ above the principal diagonal (i.e., in the positions $\left.(1,2),(2,3), \cdots,\left(n_{i}-2, n_{i}-1\right)\right)$ and zeros elsewhere. Now set

$$
C_{i}=\left[\lambda_{n_{1}+\cdots+n_{i}}\right]
$$

and

$$
X_{i}=\left[\begin{array}{c}
\lambda_{n_{1}+\cdots+n_{i-1}+1}-\lambda_{n_{1}+\cdots+n_{i}}-a_{n_{1}+\cdots+n_{i}} \\
0 \\
\cdot \\
\cdot \\
\cdot \\
0 \\
a_{n_{1}+\cdots+n_{i}-1}
\end{array}\right]
$$

where $X_{i}$ has $n_{i}-1(>1)$ rows. Let

$$
B_{i}=L_{1, n_{i}, n_{i}}^{\left(X_{i}, C_{i}\right)} A_{i}, \quad i=1, \cdots, \alpha .
$$


The eigenvalues of $B_{i}$ are $\lambda_{n_{1}+\cdots+n_{i-1}+1}, \cdots, \lambda_{n_{1}+\cdots+n_{i}}$ and the positions $(1,2), \cdots,\left(n_{i}, 1\right)$ are respectively $a_{n_{1}+\cdots+n_{i-1}+1}, \cdots, a_{n_{1}+\cdots+n_{i}}$. Now put $F=B_{1}+\cdots+B_{\alpha}$. It is clear that the eigenvalues of $F$ are $\lambda_{1}, \cdots$, $\lambda_{n_{1}+\cdots+n_{\alpha}}$, and that the $(j, \sigma(j))$ entry in $F$ is $a_{j}$ for $j=1, \cdots, n_{1}+\cdots+n_{\alpha}$.

Now, setting $n_{1}+\cdots+n_{\alpha}=p$, we can write

$$
\sigma_{\alpha+i}=(p+2 i-1, p+2 i), \quad i=1, \cdots, \beta-\alpha .
$$

Let

$$
D_{1}=\left[\begin{array}{cc}
\lambda_{p+1} & a_{p+1} \\
0 & \lambda_{p+2}
\end{array}\right], \quad Y_{1}=\left[\begin{array}{cc}
0 & 0 \\
\cdot & \cdot \\
\cdot & \cdot \\
\cdot & \cdot \\
0 & 0 \\
a_{p+2} & 0
\end{array}\right] \text { (p rows) }
$$

It can be easily seen that $F_{1}=L_{p, p+2, p+2}^{\left(Y_{1}, D_{1}\right)} F$ has eigenvalues $\lambda_{1}, \cdots, \lambda_{p+2}$ and that its $(j, \sigma(j))$ entry is $a_{j}$ for $j=1, \cdots, p+2$. Next we take

$$
D_{2}=\left[\begin{array}{cc}
\lambda_{p+3} & a_{p+3} \\
0 & \lambda_{p+4}
\end{array}\right], \quad Y_{2}=\left[\begin{array}{cc}
0 & 0 \\
\cdot & \cdot \\
\cdot & \cdot \\
\cdot & \cdot \\
0 & 0 \\
a_{p+4} & 0
\end{array}\right] \quad(p+2 \text { rows })
$$

The matrix $F_{2}=L_{p+2, p+4, p+4}^{\left(Y_{2}, D_{2}\right)} F_{1}$ has eigenvalues $\lambda_{1}, \cdots, \lambda_{p+4}$ and its $(j, \sigma(j))$ entry is $a_{j}$ for $j=1, \cdots, p+4$. Continuing in this way we arrive at a $q \times q$ matrix (where $q=n_{1}+\cdots+n_{\beta}$ ) $G$ which has eigenvalues $\lambda_{1}, \cdots$, $\lambda_{q}$ and $a_{j}$ in the position $(j, \sigma(j))$ for $j=1, \cdots, q$.

Now take

$$
E_{1}=\left[\lambda_{a+1}\right], \quad Z_{1}=\left[\begin{array}{c}
0 \\
\cdot \\
\cdot \\
\cdot \\
0 \\
a_{a+1}-\lambda_{a+1}
\end{array}\right] \text { (q rows). }
$$

The matrix $G_{1}=L_{q, q+1, q+1}^{\left(Z_{1}, E_{1}\right)} G$ has eigenvalues $\lambda_{1}, \cdots, \lambda_{q+1}$ and $a_{j}$ in the $(j, \sigma(j))$ position for $j=1, \cdots, q+1$ (we recall that the $(q, q)$ position in $G$ is not prescribed). 
Next take

$$
D_{2}=\left[\lambda_{q+2}\right], \quad Z_{2}=\left[\begin{array}{c}
0 \\
\cdot \\
\cdot \\
\cdot \\
0 \\
a_{a+2}-\lambda_{a+2} \\
0
\end{array}\right] \quad(q+1 \text { rows })
$$

Clearly the matrix $G_{2}=L_{q, q+2, q+2}^{\left(Z_{2}, D_{2}\right)} G_{1}$ has eigenvalues $\lambda_{1}, \cdots, \lambda_{q+2}$ and $a_{j}$ in the $(j, \sigma(j))$ position for $j=1, \cdots, q+2$.

There is no difficulty in seeing that continuing in this way, after a suitable number of steps, we reach a matrix which satisfies the conditions required in the theorem.

ProOF OF THEOREM 2. We may assume, without loss of generality, that the values $i_{0}, \Theta, \rho$ of the theorem are $1, \lambda_{1}, \lambda_{2}$ respectively. If the equation

$$
x^{2}-\left(\lambda_{1}+\lambda_{2}\right) x+a_{1} a_{2}+\lambda_{1} \lambda_{2}=0
$$

has a root in $\Phi$, we can find in $\Phi$ two elements $a_{11}$ and $a_{22}$ such that

$$
\left[\begin{array}{ll}
a_{11} & a_{1} \\
a_{2} & a_{22}
\end{array}\right]
$$

has eigenvalues $\lambda_{1}, \lambda_{2}$. Operating now with this $2 \times 2$ matrix as we did with the matrix $F$ appearing in the proof of the preceding theorem, we see that we can construct a matrix satisfying the requirements of the present theorem.

Proof of TheORem 3. The proof of this theorem will be by induction. For $n=2$, the theorem is obviously true. Assume the theorem is valid for $(n-1) \times(n-1)$ matrices. Following [3], there is an integer $s, 1 \leqq s \leqq n$, such that either (1) there is exactly one prescribed entry in the sth row or in the sth column, but not both, or (2) there are no prescribed entries in the sth row and sth column. Without loss of generality we may assume that $s=n$.

Case 1. This case is solved as case (i) of Theorem 3 in [3].

Case 2. The last row and column have no prescribed entries. By the induction hypothesis there exists an $(n-1) \times(n-1)$ matrix $T=\left[t_{i j}\right]$ with eigenvalues $\lambda_{1}, \cdots, \lambda_{n-1}$ and $a_{t}$ in the position $\left(i_{t}, j_{t}\right), t=1, \cdots, n-2$. 
Take

$$
C=\left[\lambda_{n}\right], \quad X=\left[\begin{array}{c}
0 \\
\cdot \\
\cdot \\
\cdot \\
0 \\
t_{i_{n-1}, j_{n-1}}-a_{n-1} \\
0 \\
\cdot \\
\cdot \\
0
\end{array}\right] \quad(n-1 \text { rows })
$$

where $t_{i_{n-1}, j_{n-1}}-a_{n-1}$ is in the $i_{n-1}$ th row of $X$. The matrix $L_{j_{n-1}, n, n}^{(X, C)} T$ has eigenvalues $\lambda_{1}, \cdots, \lambda_{n}$ and has the prescribed elements in the right positions.

REMARK. It is easily seen that in constructing the above matrices with the $L$-transform we may, in general, prescribe more entries than the ones required in our theorems. For this it is sufficient to give appropriate values to the elements of the matrices $X$ appearing in $L_{i j n}^{(X, C)}$.

In closing we mention some open problems: (1) Are the above results valid if we prescribe the characteristic polynomial (possibly with roots not in $\Phi$ ) instead of the eigenvalues? (2) What happens if we prescribe not only one diagonal but several, or entries not necessarily on a diagonal? (3) We may prescribe some entries and, instead of the eigenvalues, the singular values for example [6]. (4) We may also consider problems in which symmetry is imposed upon the matrix, etc.

The referee points out that some work related to problem (2) has been done by S. Friedland and appears in the Israel J. Math. 11 (1972), 184-189.

\section{REFERENCES}

1. H. K. Farahat and W. Ledermann, Matrices with prescribed characteristic polynomials, Proc. Edinburgh Math. Soc. 11 (1958/59), 143-146. MR 21 \#6382.

2. M. Fiedler, Matrix inequalities, Numer. Math. 9 (1966), 109-119. MR 34 \#2591.

3. D. London and $\mathrm{H}$. Minc, Eigenvalues of matrices with prescribed entries, Proc. Amer. Math. Soc. 34 (1972), 8-14; Zentralblatt Math. 215 (1971), 86.

4. L. Mirsky, Matrices with prescribed characteristic roots and diagonal elements, J. London Math. Soc. 33 (1958), 14-21. MR 19, 1034.

5. G. N. de Oliveira, Sobre matrizes estocásticas e duplamente estocásticas, Rev. Fac. Ci. Univ. Coimbra 41 (1968), 15-221. Zentralblatt Math. 212 (1971), 370. 
6. G. N. de Oliveira, Matrices with prescribed principal elements and singular values. Canad. Math. Bull. 14 (1971), 247-249.

7. - Matrices with prescribed characteristic polynomial and a prescribed submatrix, Pacific J. Math. 29 (1969), 653-661.

8. - Matrices with prescribed characteristic polynomial and a prescribed submatrix. II, Pacific J. Math. 29 (1969), 663-667.

9. - Matrices with prescribed characteristic polynomial and a prescribed submatrix. III, Monatsh. Math. 75 (1971), 441-446.

10. - Note on the function per $(\lambda I-A)$, Rev. Fac. Ci. Lisboa 13 (1971), 199-201

Current address: R. Vasco da Gama, Bloco B, $4^{\circ} \mathrm{D}$, Coimbra, Portugal 